\title{
Observations of Stably Stratified Flow through a Microscale Gap
}

\author{
Vassallo, Daniel; Krishnamurthy, Raghavendra; Menke, Robert; Fernando, Harindra J. S.
}

Published in:

Journal of the Atmospheric Sciences

Link to article, DOI:

10.1175/JAS-D-20-0087.1

Publication date:

2021

Document Version

Publisher's PDF, also known as Version of record

Link back to DTU Orbit

Citation (APA):

Vassallo, D., Krishnamurthy, R., Menke, R., \& Fernando, H. J. S. (2021). Observations of Stably Stratified Flow through a Microscale Gap. Journal of the Atmospheric Sciences, 189-208. https://doi.org/10.1175/JAS-D-200087.1

\section{General rights}

Copyright and moral rights for the publications made accessible in the public portal are retained by the authors and/or other copyright owners and it is a condition of accessing publications that users recognise and abide by the legal requirements associated with these rights.

- Users may download and print one copy of any publication from the public portal for the purpose of private study or research.

- You may not further distribute the material or use it for any profit-making activity or commercial gain

- You may freely distribute the URL identifying the publication in the public portal 
$6 \quad{ }^{1}$ Department of Biotechnology and Biomedicine, Technical University of Denmark, DK-2800

7 Kgs. Lyngby, Denmark.

8

9 * Corresponding author:

10 Lars Jelsbak, Department of Biotechnology and Biomedicine, Technical University of

11 Denmark, DK-2800 Kgs. Lyngby, Denmark. Email: lj@bio.dtu.dk. Telephone: +45 45256129

12

13 Keywords: Secondary metabolites, Pseudomonas, DAPG, Synthetic biology, biocontrol 
Fluorescent Pseudomonas spp. producing the antibiotic 2,4-diacetylphloroglucinol (DAPG) are ecologically important in the rhizosphere as they can control phytopathogens and contribute to disease suppressiveness. DAPG can also trigger a systemic resistance response in plants and stimulate root exudation and branching as well as induce plant-beneficial activities in other rhizobacteria. While studies of DAPG-producing Pseudomonas have predominantly focused on rhizosphere niches, the ecological role of DAPG as well as the distribution and dynamics

21 of DAPG-producing bacteria remains less well understood for other environments such as bulk

22 soil and grassland, where the level of DAPG producers are predicted to be low. Here, we

23 construct a whole cell biosensor for detection of DAPG and DAPG-producing bacteria from

24 environmental samples.

25 The constructed biosensor contains a phlF response module and either lacZ or lux genes as output modules assembled on a pSEVA plasmid backbone for easy transfer to different host

27 species and to enable easy future genetic modifications. We show that the sensor is highly 28 specific towards DAPG, with a sensitivity in the low nanomolar range $(>20 \mathrm{nM})$. This sensitivity is comparable to the DAPG levels identified in rhizosphere samples by chemical analysis. The biosensor enables guided isolation of DAPG-producing Pseudomonas. Using the biosensor, we probed the same grassland soil sampling site to isolate genetically related DAPG-

32 producing Pseudomonas kilonensis strains over a period of 12 months. Next, we used the 33 biosensor to determine the frequency of DAPG-producing Pseudomonads within three 34 different grassland soil sites and show that DAPG producers can constitute part of the 35 Pseudomonas population in the range of $0.35-17 \%$ at these sites. Finally, we show that the biosensor enables detection of DAPG produced by non-Pseudomonas species. 
37 Our studies show that a whole-cell biosensor for DAPG detection can facilitate isolation of 38 bacteria that produce this important secondary metabolite and provide insight into the 39 population dynamics of DAPG producers in natural grassland soil.

\section{IMPORTANCE}

42 The interest has grown for bacterial biocontrol agents as biosustainable alternatives to 43 pesticides to increase crop yields. To date, we have a broad knowledge of antimicrobial 44 compounds, such as DAPG, produced by bacteria growing in the rhizosphere surrounding plant 45 roots. However, compared to the rhizosphere niches, the ecological role of DAPG as well as 46 the distribution and dynamics of DAPG-producing bacteria remains less well understood for 47 other environments such as bulk and grassland soil. Currently, we are restricted to chemical 48 methods with detection limits and time-consuming PCR-based and probe-hybridization 49 approaches to detect DAPG and its respective producer. In this study, we have developed a 50 whole-cell biosensor, which can circumvent the labor-intensive screening process, as well as 51 increase the sensitivity at which DAPG can be detected. This enables quantification of relative 52 amounts of DAPG-producers, which in turn increases our understanding of the dynamics and 53 ecology of these producers in natural soil environments. 
55 Secondary metabolites are well-known for their potential as drugs in the medical industry. They were initially defined as dispensable, non-vital compounds to their respective producers.

57 However, recent advances in genome mining and microbial ecology are beginning to shed light on the prevalence, role and importance of these metabolites in natural environments. In soil ecology, species of fluorescent Pseudomonas isolated from naturally suppressive soils have received a great deal of attention due to their production of antimicrobial secondary metabolites, such as 2,4-diacetylphloroglucinol (DAPG). Suppression of wheat take-all disease caused by Gaeumannomyces graminis var. tritici was shown to be induced by years of crop monoculture and was associated with the root colonization of DAPG-producing fluorescent Pseudomonas (1). Moreover, production of DAPG was shown to be involved in disease control against the causative agent of tobacco black root rot, Thielaviopsis basicola (2). It has also been demonstrated that DAPG has antibacterial properties against the pathogen Erwinia carotovorum subsp. atroseptica causing soft rot of potatoes (3).

The biosynthetic gene cluster related to DAPG production in Pseudomonas comprises eight genes, phlACBDEFGH $(4,5)$. Proteins encoded by the operon of phlACBD are responsible for the synthesis of DAPG (4). The type III polyketide synthase, PhlD, initially condenses three malonyl coenzyme A molecules into phloroglucinol (PG), which is further acetylated by the enzyme complex of PhlACB into monoacetylphloroglucinol (MAPG) and DAPG (6). PhlE was identified as a putative membrane transporter with similarities to a known efflux pump in Staphylococcus aureus (4). The protein product of phlG has been described as a hydrolase that catalyses the degradation of DAPG to MAPG, thus controlling the intracellular levels of DAPG

76 (5, 7). Both $p h l F$ and $p h l H$ encode tetR-like repressors that inhibit transcription of phlACBD

77 and phlG, respectively $(5,8)$. PhlF and $\mathrm{PhlH}$ bind to operator sites located in promoters 78 upstream of the genes they regulate, thereby sterically blocking transcription. DAPG serves as 
79 the ligand for both repressors. Thus, in the presence of DAPG repression is relieved, leading to expression of phlACBD and phlG, which in turn leads to induced DAPG biosynthesis and 81 post-translational regulation $(5,8)$.

82 The complex of species belonging to the group, Pseudomonas fluorescens, has been well 83 characterized over the past three decades, due to the potential of several species to act as 84 biocontrol agents in agriculture. A recent study surveyed the phylogenetic relationship between 85166 type strains of Pseudomonas (among which 66 belonged to the $P$. fluorescens group) based on amino acid sequences of 100 gene orthologues, which further proposed the existence of 10

87 subgroups within the $P$. fluorescens clade (9). However, despite the vast diversity among $P$. 88 fluorescens, only few species belonging to two subgroups, $P$. protegens and $P$. corrugata, are 89 known to produce DAPG $(1,8)$. With the advances in genome mining and the increased 90 availability of complete genomes, the biosynthetic gene cluster phlACBDE was recently 91 identified in Pseudomonas species outside of the $P$. fluorescens group, as well as in two genera 92 of $\beta$-proteobacteria (10). However, in these cases production of DAPG has not yet been 93 demonstrated.

94 Identification and enumeration of DAPG-producing microorganisms has, to our knowledge, exclusively relied on DNA probe hybridization and PCR-based techniques. One of the most commonly employed techniques uses colony hybridization combined with a confirmatory PCR

97 to verify the presence of phlD (11). A more recent method involves culture-independent realtime PCR to quantify populations of DAPG-producing Pseudomonas in the plant rhizosphere

99 (12). A different approach is to quantify the amount of DAPG produced in situ by chemical 100 analysis. Bonsall et al. demonstrated that an optimized extraction protocol enabled quantification of DAPG isolated directly from the plant rhizosphere (13). While these 102 techniques have clear advantages, there are several drawbacks that also exist. PCR-based 103 methods are limited by DNA binding of specific primers and measures have to be taken to 

address the quantity of diverse genotypes of DAPG-producers. Chemical identification, on the other hand, is restricted by detection limits, which is directly correlated to the size of the 106 bacterial population.

107 In recent years, the synthetic biology toolbox has expanded rapidly and the use of genetically 108 engineered molecular circuits to sense molecules and conditions of interest has gained 109 increased attention. These developments have given rise to whole-cell biosensors that utilize natural regulatory systems engineered to detect metabolites and small molecules $(14,15)$.

111 Whole-cell biosensors rely on molecule recognition to either activate transcription or lift 112 repression of a reporter gene and are thus often highly sensitive with detection limits in the 113 nano- to micromolar range (16-18). Furthermore, whole-cell biosensors are tunable by addition 114 or alteration of genetic parts, which allows for higher sensitivity and increased specificity (15, 115 19). Lastly, biosensors may also be implemented as biological detectors for uncovering 116 metabolic activities in situ $(20,21)$.

117 Studies of DAPG-producing Pseudomonas species have predominantly focused on rhizosphere 118 niches, whereas the ecological role of DAPG as well as the distribution and dynamics of 119 DAPG-producing bacteria is not well understood for other environments such as bulk soil and 120 grassland. Here, we construct a whole cell biosensor as an alternative and efficient approach 121 for detection of DAPG and directed isolation of DAPG-producing bacteria from environmental 122 samples. 
125 Two whole-cell biosensors were constructed to enable specific detection of DAPG and 126 identification of DAPG-producing bacteria. Both sensors contain an identical module for 127 DAPG sensing in combination with either the lux operon or the lacZ gene as reporters (Figure 1A). The biosensor plasmids were constructed as repressor-mediated modules in an E. coli K12 DlacIZYA host. In the absence of DAPG, the TetR-like repressor protein, PhlF, binds as a dimer to the phlO operator site (8) in the promoter upstream of the reporter gene. As bioavailable

131 DAPG diffuses into the cytoplasm and binds to PhlF, the repression on the target $\mathrm{P}_{\text {phlF }}$ promoter 132 is relieved. Two reporter modules were chosen. The lux operon was used as the output reporter to obtain a highly sensitive response measured in bioluminescence units. To enable agar plate screenings for investigating the distribution and dynamics of DAPG-producing bacteria in natural microbial communities, the lacZ gene was used as the second output reporter. To ensure stable inhibition of the $\mathrm{P}_{\mathrm{phlF}}$ promoter under non-induced conditions, the phlF gene is constitutively expressed from the PlaciQ promoter. Both genetic circuits were introduced into a pSEVA plasmid background (Figure 1B) to allow for rapid and simple cloning, as well as efficient mobilization into distinct hosts by triparental mating.

To address the sensitivity and specificity of the whole-cell biosensors, microtiter bioassays were conducted. For characterization of the lux version (Figure 1C), the E. coli host with pSEVA226-DAPG lux $_{\text {was }}$ grown in LB broth containing varying concentrations of PG, MAPG or DAPG with continuous measurements of luminescence and cell density. PG and MAPG are both precursors of DAPG and thus similar compounds, allowing determination of biosensor specificity towards DAPG. For each concentration of inducer, the average luminescence was normalized to cell density over a period of 35 minutes ( 8 data points, $\mathrm{t}=172-207 \mathrm{~min}$ ). This corresponds to late exponential growth phase (Supplementary-S1). The whole-cell biosensor 
exhibited excellent sensitivity towards DAPG with a response to $20 \mathrm{nM}$ being statistically significantly higher than the negative control without added DAPG (Student's t-test, $p=0.01$ ). The biosensor did not respond to the concentrations of PG tested, but a minor response to MAPG at $>1.25 \mu \mathrm{M}$ was observed. For characterization of the lacZ version (Figure 1D), the $E$.

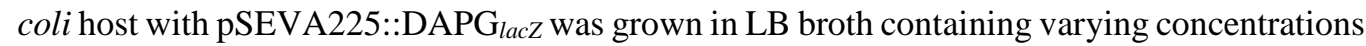
of either PG, MAPG or DAPG to allow for enzymatic expression. Subsequently, the biosensor response was determined in a $\beta$-galactosidase microtiter assay. Enzymatic activity of transcribed lac $Z$ was estimated by continuously measuring the increase in o-nitrophenol concentration over time (Supplementary-S2). The output is displayed in Miller Units (MU = $\left.\left(5000 \cdot \mathrm{OD}_{420} / \mathrm{min}\right) / \mathrm{OD}_{600}\right)$. Exposing the biosensor to $0.625 \mu \mathrm{M}$ of DAPG yielded a statistically significantly higher response compared to the negative control without added DAPG (Student's t-test, $p=0.0032$ ).

\section{Detection of DAPG production from bacterial colonies during growth on agar surfaces}

After addressing the sensitivity and specificity of the biosensor, we proceeded to utilize it in the identification of DAPG-producing bacteria. We investigated the response of the whole-cell biosensor when co-inoculated with known DAPG-producing bacteria commonly found in soil (Figure 2). The biosensor harbouring pSEVA225::DAPG lacZ $_{\text {was }}$ grown as a lawn on KBmalt agar (31) supplemented with X-gal. Inoculation of DAPG-producing cultures of $P$. protegens CHA0 and P. protegens DTU9.1 on these agar plates resulted in induction of the biosensor and production of clear blue halos surrounding the colonies after 24 hours (Figure 2A). We also constructed a $\triangle$ phlACBD mutant strain of $P$. protegens DTU9.1 by allelic replacement, in which the DAPG biosynthesis genes were deleted (Materials and Methods). As expected, the mutant strain did not elicit a response from the biosensor (Figure 2A) and did not produce DAPG detectable by LCMS analysis (Figure 2B). We note that DAPG production in 
173 Pseudomonas species has been shown to be high in growth conditions containing maltose, such 174 as the conditions used here $(8,32)$. In concordance with these findings, we did not observe 175 clear blue halos when LB agar was used (data not shown). We also tested P. putida KT2440, 176 which does not contain the DAPG biosynthetic gene cluster. Similar to the P. protegens 177 DTU9.1 $\triangle$ phlACBD mutant, $P$. putida did not elicit a response in the biosensor after 24 hours 178 of growth. However, after prolonged incubation ( $>72$ hours) of $P$. putida KT2440, a slight blue 179 colouring was detected surrounding the bacterial colony (Supplementary-S3).

180 Finally, we also explored if our setup can be used to identify DAPG production in species other 181 than Pseudomonas. Recently, the genome of C. vaccinii MWU328 was shown to contain genes 182 with high similarity to the essential genes required for DAPG biosynthesis (phlACBDE) (10). 183 We found that C. vaccinii MWU328 produced molecules that induced a response in the 184 biosensor, resulting in a blue halo around the colony. A small amount of DAPG was 185 subsequently confirmed by LCMS (Supplementary-S4). 
Next, we used our biosensor to guide the identification of DAPG-producing Pseudomonads from environmental samples. To this end, we collected soil samples from the same grassland soil site (labelled "P5") in both August 2018 and August 2019 and randomly isolated 30 fluorescent Pseudomonas strains at both time points. This site is located in Dyrehaven, which is a Danish natural reserve, thus representing a relatively unaffected, natural soil niche. Using the approach described above, all 60 isolates were screened on KBmalt agar plates supplemented with X-gal and a lawn of the biosensor harbouring pSEVA225::DAPG blue halo indicative of DAPG production was observed for one isolate from 2018 (isolate P5.21) and two isolates from 2019 (isolates P5.52 and P5.53) (Figure 3A). Subsequent LCMS analysis confirmed DAPG production in all three isolates (Supplementary-S5). For taxonomic identification of the 60 isolates, part of the housekeeping gene, $r p o D$, was sequenced for each isolate. The $r p o D$ sequences were aligned to a database of 165 Pseudomonas type strains (9). Species identification of each isolate was determined based on the highest match to the type strains using nucleotide BLAST on NCBI. The diversity of cultivable fluorescent Pseudomonas remained similar, as species of $P$. jessenii, $P$. koreensis and $P$. corrugata subgroups (as well as species of $P$. putida) were identified in the two samplings (Figure 3B). However, isolates belonging to $P$. fluorescens subgroup were only found in 2018.

The three DAPG-producing Pseudomonas were identified as P. kilonensis. This species is part of the P. corrugata subgroup of fluorescent Pseudomonas (Figure 3C, green box). Members of this subgroup are known to harbour the biosynthetic gene cluster required for DAPG production (10). To determine the phylogenetic relationship of the three $P$. kilonensis isolates compared to the $P$. corrugata subgroup, we constructed an rpoD-based bootstrap consensus tree (500 replicates) with the Neighbor-Joining method (Figure 3C). As expected, the three $P$. 
kilonensis isolates cluster together with the members of the $P$. corrugata subgroup known to produce DAPG (marked with a blue box). Taken together, these results show that genetically highly related DAPG-producing Pseudomonas can be isolated from the same grassland soil site over a 12 month period.

\section{Measuring the frequency of DAPG-producing Pseudomonads in grassland soils}

To further explore the populations and frequencies of DAPG-producing Pseudomonads in grassland soils, we sampled soil from three additional grassland sites (an area close to P5 labelled P5', P8 and P9) (Materials and methods). In total, we isolated 288 Pseudomonas strains as libraries in 96-well microplates from each of the three sites $(n=864)$. The three libraries were then screened for potential DAPG-producers by replica-plating them onto a KBmalt agar supplemented with X-gal and a lawn of the whole-cell biosensor harbouring

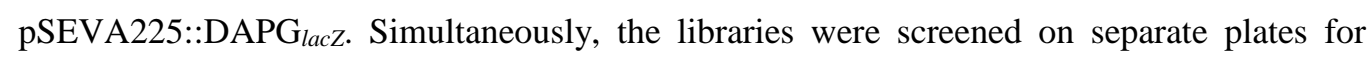
production of natural $\beta$-galactosidases, and isolates displaying a response were discarded from further analyses. Note that in this experimental setup (using replica-plating), the development of blue halos around DAPG-producing colonies took longer time than when larger aliquots of cultures were spotted on the agar plates. After 48 hours of incubation the biosensor elicited a response to six isolates from P5', five isolates from P8 and 49 isolates from P9 (Table 1). Subsequently, we analysed the colonies displaying a blue halo for the presence of $p h l D$, as well as their taxonomy by sequencing part of $r p o D$ and aligning it to the database of Pseudomonas type strains (9). For P5' and P8, one isolate from each site was confirmed to encode the polyketide synthase responsible for DAPG biosynthesis (Table 1) and both isolates were identified as P. protegens (Figure 4). For P9, the 49 isolates with a surrounding blue halo were confirmed to have phlD, where 38 of those isolates belonged to P. kilonensis, while the remaining 11 were identified as $P$. protegens (Figure 4). The false positives from P5' and P8 
237 were subsequently analysed with LC-MS (see Materials and Methods) to confirm the absence 238 of DAPG production. DAPG was not detected in any of the nine isolates.

239

240

241

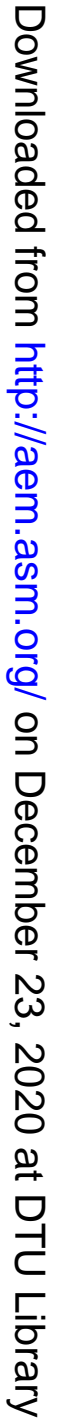


243 In this study we constructed a highly sensitive whole-cell biosensor for detection and guided 244 isolation of DAPG-producing microorganisms. Utilization of genetic circuits to detect and 245 report on the presence of small molecules is associated with advantages and disadvantages.

246 Two apparent caveats associated with the in vitro agar-based biosensor-guided identification 247 used in this study are that it is only viable for cultivable organisms and it requires conditions 248 that allow co-cultivation of the isolate of interest with the biosensor. One advantage of the 249 biosensor is that it is not restricted to a narrow range of genotypes of DAPG-producers, which 250 is a limiting factor of PCR-based approaches. Secondly, with a detection limit of $>20 \mathrm{nM}$ in 251 vitro (Figure 1C) the biosensor may have potential for use in situ to identify DAPG production 252 in specific soil niches and thus serve as a promising alternative to chemical identification of 253 DAPG, where the detection limit is in the low micromolar range (13).

254 We show that the biosensor is specific towards detection of DAPG. The biosensor did not 255 respond to PG and elicited a minor response towards MAPG. This minor response was absent 256 from its lacZ counterpart, which further demonstrates the sensitivity of the lux variant. We 257 realize that we have only used two molecules (PG and MAPG) to represent natural DAPG 258 analogues in our specificity assessment. It remains a possibility that other molecules can elicit 259 a biosensor response. In a study by Yan et al. on the PhlH transcriptional regulator, it was 260 demonstrated that multiple molecules with structural similarities to DAPG could bind to PhlH 261 and induce a response, albeit significantly lower than the response induced by DAPG (5).

262 Likewise, it was found that MAPG induced a minor response in the same study (5).

263 In order to demonstrate the biosensor response to bioavailable DAPG on agar surfaces, we 264 inoculated DAPG producers and non-producers on top of the whole-cell biosensor on agar 265 plates. As expected, a blue halo was observed around the DAPG producers. Additionally, a 266 blue colouring was absent around the non-producers after 24 hours. These findings also 
correlated with the LCMS analysis. However, a slight blue halo was observed surrounding $P$. putida after prolonged incubation (>72 hours), suggesting that one or more compounds are being secreted by this strain during late stationary phase, which interacts with PhlF, thus relieving repression of the reporter gene.

271 Subsequently, the biosensor was utilized for guided identification of DAPG-producing

272 fluorescent Pseudomonas. The isolates were selected on $1 / 4 \mathrm{~KB}^{+++}$, which has been shown to be optimal for isolation of fluorescent Pseudomonas, including DAPG producers (27). We screened 30 randomly isolated Pseudomonas from the P5 site in both 2018 and 2019. DAPG

275 producers were detected in both samplings based on a clear blue halo surrounding their colonies 276 and were identified as P. kilonensis species, which are known to produce DAPG (10). Part of 277 the rpoD gene was sequenced for all isolates and aligned to a database of Pseudomonas type strains (9), which revealed a remarkably similar diversity over a 12 month period (i.e. the same

279 Pseudomonas subgroups were sampled at both time points). However, despite the low 280 sampling depth, the species abundance appears to shift from $P$. jessenii to $P$. koreensis. From 281 an ecological point of view, it is of interest to note that the DAPG producers seem to persist 282 over time in relatively similar quantities.

283 Lastly, we sought to optimize the screening assay to a high-throughput 96-well microplate 284 format. We isolated 288 Pseudomonas species from each of three soil sites (P5', P8 and P9).

285 The sites are located in a Danish natural reserve (Dyrehaven), thus we argued that they 286 represent pristine grassland soil niches. It is worth noting that bulk soil is an extremely harsh environment with low nutrient availability, which might explain the low amount of CFU g-1 of Pseudomonas compared to rhizosphere environments (11-13). We identified 6 and 5 isolates with blue halos around their colonies in P5' and P8, respectively. Yet, only one isolate from 290 each site was confirmed to encode the polyketide synthase responsible for DAPG production. 291 Picard et al. isolated 156 Pseudomonas from bulk soil, but no DAPG producers were identified, 
although DAPG producers were isolated at a later stage from roots of maize plants grown in the same soil (33). It was speculated that DAPG-producing Pseudomonas are present in bulk 294 soil in quantities $<2.6 \cdot 10^{2} \mathrm{CFU} \mathrm{g}^{-1}$, which is comparable to the findings obtained in our study 295 of grassland soil at P5' $\left(6 \times 10^{1} \mathrm{~g}^{-1}\right)$ and P8 $\left(2 \times 10^{1} \mathrm{~g}^{-1}\right)$ (Table 1). In P9, on the other hand, $29617 \%$ of the isolates displayed a blue halo around their colonies and the presence of phlD was confirmed for all isolates. However, during the course of our study it became apparent that a deer-feeding site was located near the sampling site, with wheat being the main feed. This could potentially explain the high frequency of DAPG producers in P9, as DAPG-producing Pseudomonas, which are known to be associated with the roots of wheat (1), might have translocated into the soil surrounding the feeding site due to animal activities.

The false positive isolates from the high-throughput screening (i.e. isolates that resulted in a biosensor response without the presence of DAPG biosynthesis genes) could potentially produce compounds similar to those made by $P$. putida (as described above), which interfere with PhlF. The phlF gene encoded by the biosensor was cloned from P. protegens CHA0 (22), where it naturally functions as a transcriptional repressor of phlACBD (8). The false positives identified in our screen may secrete yet unknown secondary metabolites that interact with the PhlF repressor, thus inducing biosynthesis of DAPG. This finding highlights the possibility for microbe-microbe interactions in situ leading to induced DAPG production by adjacent non-

310 producers. Interestingly, we found two isolates belonging to the $P$. putida group in the high311 throughput screening, which may indicate that certain species of this group produce molecules 312 that can induce expression of DAPG. Surprisingly, we also identified isolates of $P$. koreensis and $P$. mandelii that elicit a response from the biosensor, which further enhances the potential of yet unexplored microbe-microbe interactions that could be addressed in future studies.

315 In conclusion, this study demonstrates the use of an engineered whole-cell biosensor for guided 316 identification of DAPG-producing microorganisms. This approach surpasses the limits of 
317 previous PCR-based and chemical identification methods, although future optimization to 318 further increase sensitivity and reduce unexpected response to false positives might be required.

319

320

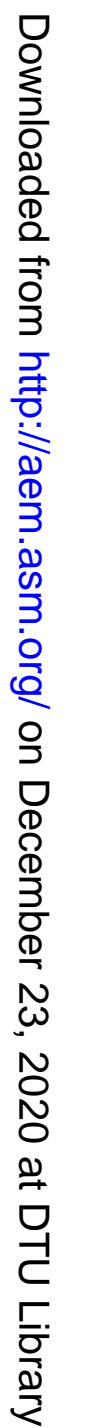




\section{Strains, media and growth conditions}

323 Plasmid cloning and genetic circuit characterization were performed in Escherichia coli K12

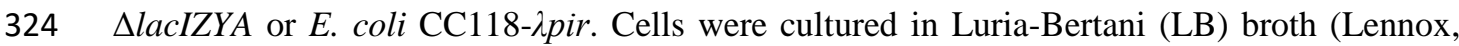
325 Merck, St. Louis, MO, USA) with appropriate antibiotics. The antibiotic concentration used 326 was $25 \mu \mathrm{g} \mathrm{ml}^{-1}$ for kanamycin, $10 \mu \mathrm{g} \mathrm{ml}^{-1}$ for chloramphenicol and $8 \mu \mathrm{g} \mathrm{ml} \mathrm{m}^{-1}$ for tetracycline.

327 The engineered whole-cell biosensor was cultured by inoculating a single colony in 5 ml LB 328 broth supplemented with kanamycin and incubating overnight at $37^{\circ} \mathrm{C}$ with shaking (200 rpm).

329 For characterization of the biosensor response to DAPG-producers, control strains were 330 routinely cultured by inoculating a single colony in $5 \mathrm{ml} \mathrm{LB}$ broth and incubating overnight at $33130^{\circ} \mathrm{C}$ with shaking $(200 \mathrm{rpm})$. Control strains include: Pseudomonas putida KT2440, 332 Pseudomonas protegens DTU9.1 (previously isolated by our group), Pseudomonas protegens 333 DTU9.1 $\triangle$ phlACBD (see below) and Chromobacterium vaccinii MWU328.

\section{Plasmid circuit construction}

Plasmid construction and DNA manipulation was performed following standard molecular biology techniques. The strain E. coli K12 $\Delta$ lacIZYA was transformed with all plasmid constructs by chemical transformation. The plasmid pAJM847 (Accession: MH101727.1), comprising the $\mathrm{P}_{\text {lacIQ }}$ promoter, phlF, the induction operon terminator (IOT) and the $\mathrm{P}_{\text {phlF }}$ promoter, was a kind gift from Christopher Voigt (22). The genetic circuit from pAJM847 was re-organized into pSEVA225T (Accession: KC847299.1) (23) to obtain the DAPG biosensor.

342 To this end, the fragment containing the $\mathrm{P}_{\text {laciQ }}$ promoter, phlF and the induction operon 343 terminator was PCR amplified with AvrII and EcoRI overhangs (PlacI_AvrII_fw: 5’- 
TGCTTAGAatTCAgCGAgGAAGCACC). Purified PCR product was digested with appropriate restriction enzymes and inserted in pSEVA225T to yield pSEVA225::PlaciQ-phlF.

347 Subsequently, the fragment containing the $\mathrm{P}_{\text {phIF }}$ promoter was PCR amplified with EcoRI and 348 HindIII overhangs (PphlF_EcoRI_fw: 5'-TAAGCAGAATTCCGACGTACGGTGG, 349 PphlF_HindIII_rev: 5’-TGCTTAAAGCTTTATTTCCCCTCTTTCTCTAG). Purified PCR 350 product was restriction-digested and inserted in pSEVA225::PlaciQ-phlF to yield the lacZ 351 version of the DAPG biosensor (pSEVA225::DAPGlacz). The lux operon (luxCDABE from $P$. 352 luminescens) was PCR amplified from pUC18-mini-TN7T-Gm-lux with HindIII and SpeI 353 overhangs (Lux_HindIII_fw: 5'-GAATTCAAGCTTATGACTAAAAAAATTTCATTC, 354 Lux_SpeI_rev: 5'-GAATTCACTAGTAGGATATCAACTATCAAAC). Purified PCR 355 product was restriction-digested and inserted in pSEVA225::DAPG lacz to yield the lux version 356 (pSEVA226::DAPGlux).

\section{Deletion of phlACBD by allelic replacement}

359 To abolish DAPG production in P. protegens DTU9.1, the biosynthesis genes, phlACBD, were 360 deleted by allelic replacement according to Hmelo et al. (24). In short, DNA fragments directly 361 upstream of phlA (Up_F: 5'-ATCCCGTCTAGACAGAGATTTCGCAGTAAAAAG, Up_R: 5’-GGCGGGACAGGCACAGGCAGTCACATTTCCTCTTGATTCCATTCTTTTC) and 363 directly downstream of phlD (Down_F: 5'-TGTGACTGCCTGTGCCTG, Down_R: 5'364 ATCCGGGAGCTCTTGAAAGCCGCCAGCC) were PCR amplified. The fragments were 365 joined by splicing-by-overlap extension PCR with XbaI and SacI overhangs using primers 366 Up_F and Down_R. The purified PCR product was restriction-digested and inserted in pNJ1 367 (25). The resulting plasmid was mobilized into P. protegens DTU9.1 via triparental mating 368 with E. coli HB101 harbouring the helper plasmid pRK600. Merodiploid transconjugants were 
initially selected on Pseudomonas Isolation Agar (PIA, Merck, St. Louis, MO, USA) supplemented with $50 \mu \mathrm{g} \mathrm{ml}^{-1}$ tetracycline. A second selection was performed on NSLB agar

371 (10 $\mathrm{g} \mathrm{l}^{-1}$ tryptone, $5 \mathrm{~g} \mathrm{l}^{-1}$ yeast extract, $15 \mathrm{~g} \mathrm{l}^{-1}$ Bacto agar) with 15\% sucrose. Candidates for 372 successful deletion were confirmed by PCR and verified by Sanger sequencing at Eurofins 373 Genomics.

\section{Luminescence dose/response microplate assay}

376 Overnight cultures of the whole-cell biosensor harbouring pSEVA226-DAPGlux were prepared in six biological replicates as described above. 96-well black, clear bottom microplates (In

378 Vitro, Denmark) were prepared with LB broth supplemented with kanamycin and varying concentrations of PG, MAPG or DAPG (0, 0.005, 0.01, 0.02, 0.039, 0.078, 0.156, 0.3125, $0.625,1.25,2.5,5,7.5,10$ and $15 \mu \mathrm{M})$. The overnight cultures were diluted to inoculate the microplates to an initial $\mathrm{OD}_{600}=0.01$. The plates were sealed with a semipermeable membrane (Breathe-Easy, Merck, St. Louis, MO, USA) and incubated in a Cytation5 microplate reader for 5 hours at $37^{\circ} \mathrm{C}$ with shaking $(600 \mathrm{rpm})$ with continuous measurements of luminescence and absorbance at $\mathrm{OD}_{600}$. This data and any other microplate assay data was collected with the Gen5 2.07 software and exported to Excel 2016 and GraphPad for data analysis.

\section{B-galactosidase dose/response microplate assay}

Overnight cultures of the whole-cell biosensor harbouring pSEVA225-DAPG lacz were prepared in triplicate as described above. Transparent 96-well microplates (TPP, Merck, St. Louis, MO, USA) were prepared with LB broth supplemented with kanamycin and varying concentrations of PG, MAPG or DAPG $(0,0.625,1.25,2.5,5,7.5,10$ and $15 \mu \mathrm{M})$. The overnight cultures were diluted to inoculate the microplates to an initial $\mathrm{OD}_{600}=0.01$. Cultures 
were grown for 3 hours at $37^{\circ} \mathrm{C}$ with shaking ( $600 \mathrm{rpm}$ ), followed by measuring the end-point absorbance at $\mathrm{OD}_{600}$. Subsequently, $20 \mu \mathrm{l}$ from each well was transferred to new transparent 96-well microplates and mixed with $80 \mu$ permeabilization buffer $\left(0.1 \mathrm{M} \mathrm{Na}_{2} \mathrm{HPO}_{4}, 0.02 \mathrm{M}\right.$ $\mathrm{KCl}, 0.002 \mathrm{M} \mathrm{MgSO}_{4}, 0.8 \mathrm{mg} \mathrm{ml}^{-1}$ hexadecyltrimethylammonium bromide, $0.4 \mathrm{mg} \mathrm{ml}^{-1}$ sodium deoxycholate, $5.4 \mu \mathrm{ml}^{-1} \beta$-mercaptoethanol) (26). The plates were incubated at $30^{\circ} \mathrm{C}$ with shaking (600 rpm) for 30 minutes to facilitate cell lysis. Next, $28 \mu$ lysed cell culture from each well was transferred to 96-well black microplates and mixed with $172 \mu \mathrm{l}$ substrate solution (0.06 $\quad \mathrm{M} \quad \mathrm{Na}_{2} \mathrm{HPO}_{4}, \quad 0.04 \quad \mathrm{M} \quad \mathrm{NaH}_{2} \mathrm{PO} 4, \quad 1 \quad \mathrm{mg}^{-1} \quad \mathrm{ml}^{-1}$ o-nitro-phenyl- $\beta$-D401 galactopyranoside, $2.7 \mu \mathrm{ml}^{-1} \beta$-mercaptoethanol) (26). The plates were sealed with a semipermeable membrane (Breathe-Easy, Merck, St. Louis, MO, USA) and incubated in a Cytation 5 microplate reader for 16 hours at $37^{\circ} \mathrm{C}$ with shaking (600 rpm) with continuous measurements of absorbance at $\mathrm{OD}_{420}$. Data was collected and analysed, as mentioned above.

\section{Detecting DAPG from bacterial cultures grown on agar surfaces}

407 Overnight cultures of the whole-cell biosensor harbouring pSEVA225-DAPGlacz and the 408 control strains $P$. putida KT2440, P. protegens DTU9.1, P. protegens DTU9.1 $\triangle$ phlACBD and 409 C. vaccinii MWU328 were prepared as described above. The biosensor was normalized to $410 \mathrm{OD}_{600}=1.0$ and spread on King's agar B supplemented with malt extract (KBmalt) $\left(20 \mathrm{~g} \mathrm{l}^{-1}\right.$ 411 Proteose peptone No. 3, $1.5 \mathrm{~g} \mathrm{l}^{-1} \mathrm{~K}_{2} \mathrm{HPO}_{4}, 1.5 \mathrm{~g} \mathrm{l}^{-1} \mathrm{MgSO}_{4}, 7.5 \mathrm{~g} \mathrm{l}^{-1}$ malt extract, $10 \mathrm{ml} \mathrm{l}$ 412 glycerol and $20 \mathrm{~g} \mathrm{l}^{-1}$ Bacto agar) supplemented with $25 \mathrm{\mu g} \mathrm{ml}^{-1}$ 5-bromo-4-chloro-3-indolyl- $\beta$ 413 D-galactopyranoside (X-gal, Thermo Fisher Scientific). Overnight cultures of the control 414 strains were normalized to $\mathrm{OD}_{600}=1.0$ and inoculated as $20 \mu \mathrm{l}$ spots on the agar plates 415 containing the biosensor. Agar plates were incubated at $30^{\circ} \mathrm{C}$ for $24-96$ hours. Plates were inspected for blue halos surrounding the bacterial spots every 24 hours. 
418 Overnight cultures of the control strains $P$. putida KT2440, P. protegens DTU9.1, P. protegens

419 DTU9.1 $\triangle$ phlACBD and C. vaccinii MWU328 were prepared as described above. The cultures 420 were normalized to $\mathrm{OD}_{600}=1.0$, inoculated as $20 \mu \mathrm{l}$ spots on KBmalt and malt agar plates and 421 incubated at $30^{\circ} \mathrm{C}$ for 24 hours. An agar plug ( $6 \mathrm{~mm}$ diameter) of the bacterial culture was 422 transferred to a vial and extracted with $1 \mathrm{ml}$ of isopropanol:ethyl acetate (1:3, v/v), containing $4231 \%$ formic acid, under ultrasonication for $60 \mathrm{~min}$. The extracts were then transferred to new 424 vials, evaporated under $\mathrm{N}_{2}$, and re-dissolved in $200 \mu \mathrm{l}$ of methanol for further sonication over $42515 \mathrm{~min}$. After centrifugation at $13400 \mathrm{rpm}$ for $3 \mathrm{~min}$, the supernatants were transferred to HPLC 426 vials and subjected to ultrahigh-performance liquid chromatography-high resolution electrospray ionization mass spectrometry (UHPLC-HRESIMS) analysis. UHPLC-HRESIMS was performed on an Agilent Infinity 1290 UHPLC system equipped with a diode array (DAD)

429 detector. UV-visible spectra were recorded from 190 to $640 \mathrm{~nm}$. Liquid chromatography of 1 $430 \mu$ l extract was carried out using an Agilent Poroshell 120 phenyl-hexyl column $(2.1 \times 150 \mathrm{~mm}$, $431 \quad 1.9 \mu \mathrm{m}$ ) at $60^{\circ} \mathrm{C}$ using acetonitrile and $\mathrm{H}_{2} \mathrm{O}$, both containing $0.02 \mathrm{M}$ formic acid, as mobile 432 phases. Initially, a linear gradient of $10 \%$ acetonitrile/ $\mathrm{H}_{2} \mathrm{O}$ to $100 \%$ acetonitrile over 10 minutes 433 was employed, followed by isocratic wash of $100 \%$ acetonitrile for 2 minutes. The gradient 434 was returned to $10 \%$ acetonitrile/ $\mathrm{H}_{2} \mathrm{O}$ in 0.1 minute and finally isocratic condition of $10 \%$ 435 acetonitrile $/ \mathrm{H}_{2} \mathrm{O}$ for 1.9 minutes, all at a flow rate of $0.35 \mathrm{ml} \mathrm{min}{ }^{-1}$. Mass-spectrometry 436 detection was performed in positive ionization on an Agilent 6545 QTOF MS equipped with 437 an Agilent Dual Jet Stream electrospray ion source with a drying gas temperature of $250^{\circ} \mathrm{C}$, 438 drying gas flow of $8 \mathrm{l} \mathrm{min}^{-1}$, sheath gas temperature of $300^{\circ} \mathrm{C}$ and sheath gas flow of $12 \mathrm{~L} \mathrm{~min}^{-}$

439 . Capillary voltage was set to $4000 \mathrm{~V}$ and nozzle voltage to $500 \mathrm{~V}$. Mass-spec data analysis 440 and processing were performed using Agilent MassHunter Qualitative Analysis B.07.00. 
443 Three sites of undisturbed grassland were chosen (P5, 55078'88’N, 12055'83”E; P8, 444 55079’52”N, 12058’06”E; P9, 55079’12”N, 12057’51”E). Soil was collected approximately 10 445 centimetres below the grass surface. Five grams of soil were suspended in $30 \mathrm{ml}$ of sterile water 446 and shaken vigorously for $1 \mathrm{~min}$ on a Vortex mixer. In order to isolate fluorescent 447 Pseudomonas the samples were serially diluted and plated onto $1 / 4 \mathrm{~KB}^{+++}\left(7.5 \mathrm{~g} \mathrm{l}^{-1}\right.$ King's agar B, $10 \mathrm{ml} \mathrm{l}^{-1}$ glycerol, $7.5 \mathrm{~g} \mathrm{l}^{-1}$ Bacto agar) supplemented with $100 \mu \mathrm{g} \mathrm{ml}^{-1}$ cycloheximide, 13 $\mu \mathrm{g} \mathrm{ml} \mathrm{l}^{-1}$ chloramphenicol and $40 \mu \mathrm{g} \mathrm{ml}^{-1}$ ampicillin, as previously described (27). Agar plates

450 were incubated at $30^{\circ} \mathrm{C}$ for 48 hours. Fluorescent colonies were identified under UV light and 451 re-streaked on LB agar plates. Species identification of the soil isolates was performed by PCR, 452 amplifying part of the rpoD gene with primers (PsEG30F: 5'-ATYGAAATCGCCAARCG, 453 PsEG790R: 5'-CGGTTGATKTCCTTGA) (28). PCR products were purified, sequenced and aligned to a database of 166 known type strains of Pseudomonas (9).

\section{Biosensor-guided identification of DAPG-producers from grassland soil}

457 Thirty fluorescent Pseudomonas were randomly selected from sample site P5 both in 2018 and 4582019 as described above. Isolates were cultured overnight in $\mathrm{LB}$ broth at $30^{\circ} \mathrm{C}$ with shaking 459 (200 rpm). An overnight culture of the biosensor harbouring pSEVA225-DAPGlacz was 460 normalized to $\mathrm{OD}_{600}=1.0$ and spread on KBmalt plates supplemented with $25 \mu \mathrm{g} \mathrm{ml}^{-1} \mathrm{X}$-gal. 461 Overnight cultures of the Pseudomonas isolates were inoculated on the agar plates as $20 \mu \mathrm{l}$ 462 spots. Plates were incubated at $30^{\circ} \mathrm{C}$ for $24-48$ hours. Plates were inspected for blue halos 463 surrounding the bacterial spots every 24 hours. The DAPG-producing isolates were identified 464 by PCR-based species identification, as described above. The phylogenetic relationship 465 between the DAPG-producing isolates was determined by analysing a phylogenetic tree with 466 representatives of each $P$. fluorescens subgroup (9). In short, the PCR amplified part of the 
rpoD genes were Sanger sequenced and aligned using the MUSCLE algorithm, followed by construction of a bootstrap consensus tree (500 replicates) by the neighbour-joining method in MEGA X (29).

470

471

\section{High-throughput screening for DAPG-producers in grassland soil}

472 In 2019, 288 fluorescent Pseudomonas were randomly selected from three sample sites (P5', 473 55078'78’ N, 12056’07”E; P8 and P9 - see coordinates above). Fluorescent colonies were 474 streaked on LB agar OmniTray ${ }^{\mathrm{TM}}$ (Nunc $^{\mathrm{TM}}$, Nalge Nunc International, Rochester, NY, USA) 475 and incubated for 24 hours at $30^{\circ} \mathrm{C}$. Isolates were cultured in transparent 96-well microplates 476 in terrific broth (TB; $12 \mathrm{~g} \mathrm{l}^{-1}$ tryptone, $24 \mathrm{~g} \mathrm{l}^{-1}$ yeast extract, $0.17 \mathrm{M} \mathrm{KH}_{2} \mathrm{PO}_{4}, 0.72 \mathrm{M} \mathrm{K}_{2} \mathrm{HPO}_{4}$ 477 and $5 \mathrm{ml} \mathrm{l}^{-1}$ glycerol). An overnight culture of the biosensor harbouring pSEVA225-DAPG acz 478 was normalized to $\mathrm{OD}_{600}=0.5$ and spread on KBmalt OmniTrays supplemented with $50 \mu \mathrm{g}$ $479 \mathrm{ml}^{-1} \mathrm{X}$-gal. The Pseudomonas isolates were inoculated on the OmniTrays with sterile 480 replicators. The OmniTrays were incubated at $30^{\circ} \mathrm{C}$ for 48 hours and inspected for blue halos 481 surrounding the Pseudomonas colonies. Candidate isolates exhibiting a blue halo were 482 screened with PCR for the presence of phlD with primers (B2BF: 5'483 ACCCACCGCAGCATCGTTTATGAGC, BPR4: 484 CCGCCGgtAtGgAAgAtGAAAAAGTC) (30). Moreover, rpoD was amplified from 485 candidate colonies for species identification with primers PsEG30F and PsEG790R.

487 Data availability

488 All data are included in the article and the supplemental material. Plasmid constructs and 489 bacterial strains, including Pseudomonas isolates, will be made available by the corresponding 490 author upon request. 


\section{Acknowledgements}

492 We would like to thank Prof. Victor de Lorenzo for providing the pSEVA plasmid used as

493 vector background for the biosensor. Secondly, we thank Prof. Christopher Voigt for providing

494 the plasmid pAJM847. Moreover, we want to thank Pavelas Sazinas for advice on phylogenetic

495 analysis, as well as Susanne Koefoed for technical assistance. We thank the members of the

496 Centre for Microbial Secondary Metabolites (CeMiSt) for discussions.

497

498

499 Funding

500 This study was funded by the Danish National Research Foundation (DNRF137) for the

501 Centre for Microbial Secondary Metabolites. 
503 1. Raaijmakers JM, Weller DM. 1998. Natural Plant Protection by 2,4-

504 Diacetylphloroglucinol-Producing Pseudomonas spp. in Take-All Decline Soils. Mol

$505 \quad$ Plant-Microbe Interact 11:144-152.

506

2. Ramette A, Moënne-Loccoz Y, Défago G. 2003. Prevalence of fluorescent pseudomonads producing antifungal phloroglucinols and/or hydrogen cyanide in soils naturally suppressive or conducive to tobacco black root rot. FEMS Microbiol Ecol $44: 35-43$.

3. Cronin D, Moënne-Loccoz Y, Fenton A, Dunne C, Dowling DN, O’Gara F. 2006. Ecological interaction of a biocontrol Pseudomonas fluorescens strain producing 2,4diacetylphloroglucinol with the soft rot potato pathogen Erwinia carotovora subsp. atroseptica. FEMS Microbiol Ecol 23:95-106.

4. Bangera MG, Thomashow LS. 1999. Identification and Characterization of a Gene Cluster for Synthesis of the Polyketide Antibiotic 2,4-Diacetylphloroglucinol from Pseudomonas fluorescens Q2-87. J Bacteriol 181:3155-3163.

5. Yan X, Yang R, Zhao R-X, Han J-T, Jia W-J, Li D-Y, Wang Y, Zhang N, Wu Y, Zhang L-Q, He Y-X. 2017. Transcriptional Regulator PhlH Modulates 2,4-

Diacetylphloroglucinol Biosynthesis in Response to the Biosynthetic Intermediate and End Product. Appl Environ Microbiol 83.

6. Yang F, Cao Y. 2012. Biosynthesis of phloroglucinol compounds in microorganisms—review. Appl Microbiol Biotechnol 93:487-495.

7. Bottiglieri M, Keel C. 2006. Characterization of PhlG, a hydrolase that specifically degrades the antifungal compound 2,4-diacetylphloroglucinol in the biocontrol agent 
Pseudomonas fluorescens CHA0. Appl Environ Microbiol 72:418-27.

526

8. Abbas A, Morrissey JP, Marquez PC, Sheehan MM, Delany IR, O’Gara F. 2002. Characterization of interactions between the transcriptional repressor PhlF and its binding site at the phlA promoter in Pseudomonas fluorescens F113. J Bacteriol 184:3008-16.

9. Hesse C, Schulz F, Bull CT, Shaffer BT, Yan Q, Shapiro N, Hassan KA, Varghese N, Elbourne LDH, Paulsen IT, Kyrpides N, Woyke T, Loper JE. 2018. Genome-based evolutionary history of Pseudomonas spp. Environ Microbiol 20:2142-2159.

10. Almario J, Bruto M, Vacheron J, Prigent-Combaret C, Moënne-Loccoz Y, Muller D. 2017. Distribution of 2,4-Diacetylphloroglucinol Biosynthetic Genes among the Pseudomonas spp. Reveals Unexpected Polyphyletism. Front Microbiol 8:1218.

11. Raaijmakers JM, Weller DM, Thomashow LS. 1997. Frequency of AntibioticProducing Pseudomonas spp. in Natural Environments. Appl Environ Microbiol 63:881-7.

12. Mavrodi O V, Mavrodi D V, Thomashow LS, Weller DM. 2007. Quantification of 2,4diacetylphloroglucinol-producing Pseudomonas fluorescens strains in the plant rhizosphere by real-time PCR. Appl Environ Microbiol 73:5531-8.

13. Bonsall RF, Weller DM, Thomashow LS. 1997. Quantification of 2,4Diacetylphloroglucinol Produced by Fluorescent Pseudomonas spp. In Vitro and in the Rhizosphere of Wheat. Appl Environ Microbiol 63:951-5.

14. Renella G, Giagnoni L. 2016. Light dazzles from the black box: whole-cell biosensors are ready to inform on fundamental soil biological processes. Chem Biol Technol Agric 3:8. 
15. Wan X, Ho TYH, Wang B. 2019. Engineering Prokaryote Synthetic Biology Biosensors, p. 1-37. In Handbook of Cell Biosensors. Springer International Publishing, Cham.

16. Kim HJ, Lim JW, Jeong H, Lee S-J, Lee D-W, Kim T, Lee SJ. 2016. Development of

17. Wang B, Barahona M, Buck M. 2015. Amplification of small molecule-inducible gene a highly specific and sensitive cadmium and lead microbial biosensor using synthetic CadC-T7 genetic circuitry. Biosens Bioelectron 79:701-708. expression via tuning of intracellular receptor densities. Nucleic Acids Res 43:19551964.

18. Hooshangi S, Thiberge S, Weiss R. 2005. Ultrasensitivity and noise propagation in a synthetic transcriptional cascade. Proc Natl Acad Sci U S A 102:3581-6.

19. Wang B, Barahona M, Buck M. 2014. Engineering modular and tunable genetic amplifiers for scaling transcriptional signals in cascaded gene networks. Nucleic Acids Res 42:9484-9492.

20. Burmølle M, Hansen LH, Sørensen SJ. 2005. Use of a Whole-Cell Biosensor and Flow Cytometry to Detect AHL Production by an Indigenous Soil Community During Decomposition of Litter. Microb Ecol 50:221-229.

21. Mimee M, Nadeau P, Hayward A, Carim S, Flanagan S, Jerger L, Collins J, McDonnell S, Swartwout R, Citorik RJ, Bulović V, Langer R, Traverso G, Chandrakasan AP, Lu TK. 2018. An ingestible bacterial-electronic system to monitor gastrointestinal health. Science 360:915-918.

22. Meyer AJ, Segall-Shapiro TH, Glassey E, Zhang J, Voigt CA. 2019. Escherichia coli “Marionette” strains with 12 highly optimized small-molecule sensors. Nat Chem Biol 
572

23. Silva-Rocha R, Martínez-García E, Calles B, Chavarría M, Arce-Rodríguez A, de las Heras A, Páez-Espino AD, Durante-Rodríguez G, Kim J, Nikel PI, Platero R, de Lorenzo V. 2013. The Standard European Vector Architecture (SEVA): a coherent platform for the analysis and deployment of complex prokaryotic phenotypes. Nucleic Acids Res 41:D666-D675.

24. Hmelo LR, Borlee BR, Almblad H, Love ME, Randall TE, Tseng BS, Lin C, Irie Y, Storek KM, Yang JJ, Siehnel RJ, Howell PL, Singh PK, Tolker-Nielsen T, Parsek MR, Schweizer HP, Harrison JJ. 2015. Precision-engineering the Pseudomonas aeruginosa genome with two-step allelic exchange. Nat Protoc 10:1820-1841.

25. Yang L, Hengzhuang W, Wu H, Damkiær S, Jochumsen N, Song Z, Givskov M, Høiby N, Molin S. 2012. Polysaccharides serve as scaffold of biofilms formed by mucoid Pseudomonas aeruginosa. FEMS Immunol Med Microbiol 65:366-376.

26. Zhang X, Bremer H. 1995. Control of the Escherichia coli rrnB P1 promoter strength by ppGpp. J Biol Chem 270:11181-9.

27. Landa BB, De Werd HAE, McSpadden Gardener BB, Weller DM. 2002. Comparison of three methods for monitoring populations of different genotypes of 2,4diacetylphloroglucinol-producing Pseudomonas fluorescens in the rhizosphere. Phytopathology 92:129-137.

28. Mulet M, Bennasar A, Lalucat J, García-Valdés E. 2009. An rpoD-based PCR procedure for the identification of Pseudomonas species and for their detection in environmental samples. Mol Cell Probes 23:140-147.

29. Kumar S, Nei M, Dudley J, Tamura K. 2008. MEGA: A biologist-centric software for 
evolutionary analysis of DNA and protein sequences. Brief Bioinform 9:299-306.

595 30. McSpadden Gardener BB, Mavrodi D V., Thomashow LS, Weller DM. 2001. A Rapid

596 Polymerase Chain Reaction-Based Assay Characterizing Rhizosphere Populations of

597 2,4-Diacetylphloroglucinol-Producing Bacteria. Phytopathology 91:44-54.

598 31. Baehler E, Bottiglieri M, Péchy-Tarr M, Maurhofer M, Keel C. 2005. Use of green

599 fluorescent protein-based reporters to monitor balanced production of antifungal

600 compounds in the biocontrol agent Pseudomonas fluorescens CHA0. J Appl Microbiol

$601 \quad 99: 24-38$.

602 32. Maurhofer M, Keel C, Haas D, Défago G. 1994. Pyoluteorin production by

603 Pseudomonas fluorescens strain CHA0 is involved in the suppression of Pythium

604 damping-off of cress but not of cucumber. Eur J Plant Pathol 100:221-232.

605 33. Picard C, Di Cello F, Ventura M, Fani R, Guckert A. 2000. Frequency and biodiversity

606 of 2,4-diacetylphloroglucinol-producing bacteria isolated from the maize rhizosphere

607 at different stages of plant growth. Appl Environ Microbiol 66:948-55.

608

609 
Figure 1. Design of highly specific and sensitive whole-cell biosensors for DAPG detection.

611 A) Schematic illustration of the genetic circuit representing the DAPG biosensors with varying

612 reporter modules. B) Map of the biosensor present on a pSEVA plasmid background with an

613 RK2 replicon (orange), origin of transfer (dark grey) and a kanamycin resistance gene (light

614 green). Terminators (T0, T1 and IOT - dark red) C) The response of the whole-cell biosensor

615 harbouring pSEVA226-DAPG

616 in luminescence per $\mathrm{OD}_{600}$. D) The response of the whole-cell biosensor harbouring

617 pSEVA225-DAPG lacz to DAPG and similar molecules (PG and MAPG) measured in Miller

618 Units.

619

620 Figure 2. Detection of DAPG produced by bacterial colonies grown on agar surfaces. A)

621 Four fluorescent Pseudomonas were grown on a lawn of the biosensor with 622 pSEVA225::DAPG ${ }_{\text {lacz }}$ on KBmalt media supplemented with X-gal. Wildtype P. protegens

623 CHA0 and DTU9.1 known to produce DAPG elicited a biosensor response after 24 hours,

624 whereas the negative controls $P$. putida KT2440 and a $\triangle$ phlACBD mutant of $P$. protegens

625 DTU9.1 did not. B) Extracted ion chromatograms (EIC) for DAPG ( $\mathrm{m} / \mathrm{z} 211.0601 \pm 5 \mathrm{ppm})$ of

626 the four Pseudomonas extracts confirm the production of DAPG after 24 hours by P. protegens

627 CHA0 and DTU9.1.

628

629 Figure 3. Examination of fluorescent Pseudomonas from grassland soil for DAPG

630 production. A) 30 fluorescent Pseudomonas isolated from grassland soil in 2018/2019 were

631 grown on a lawn of the biosensor with pSEVA225::DAPG lacz $_{\text {on KBmalt media supplemented }}$

632 with X-gal. The biosensor responded to one isolate from 2018 and two from 2019. B) Part of

633 the $r p o D$ gene was sequenced and aligned to a database of 165 type strains of Pseudomonas.

634 The three isolates eliciting a response from the biosensor were identified as $P$. kilonensis, which 
is part of the $P$. corrugata subgroup (9). C) An rpoD-based Neighbor-Joining tree representing the phylogenetic relationship of the three $P$. kilonensis isolates to the $P$. corrugata subgroup. P. protegens CHA0 was included as outlier. A bootstrap consensus tree (500 replicates) of the

638 rpoD PCR products was constructed via the Neighbor-Joining method. The bootstrap 639 percentage values are depicted next to each branching point. The $P$. corrugata subgroup is 640 depicted in a green box, whereas the species of this subgroup known to produce DAPG are 641 placed in a blue box, along with the three soil isolates.

642

643 Figure 4. Taxonomic identification of isolates with a surrounding blue halo in the high-

644 throughput screening assay. For each isolate, part of the $r p o D$ gene was sequenced and 645 aligned to a database of 165 type strains of Pseudomonas. In both P5' and P8, one isolate was 646 identified as $P$. protegens, whereas the remaining isolates belonged to either the $P$. putida group 647 or $P$. koreensis and $P$. mandelii subgroups of $P$. fluorescens, according to Hesse et al. (9). In $648 \mathrm{P} 9$, the $r p o D$ gene of 49 isolates displaying a blue halo was sequenced. Only species of $P$. 649 kilonensis and $P$. protegens were identified. 
TABLE 1. Frequencies of DAPG producers in natural soil microbiomes

\begin{tabular}{|c|c|c|c|c|c|}
\hline & \multirow{2}{*}{$\begin{array}{c}\text { Total CFU/g } \\
\text { soil }^{a}\end{array}$} & \multicolumn{2}{|c|}{ Blue halo } & \multicolumn{2}{|c|}{ phlD PCR verified } \\
\hline & & Count & Percentage $^{b}$ & Count & $\mathrm{CFU} / \mathrm{g}$ soil $^{\mathrm{c}}$ \\
\hline$\overline{P 5}$ & $1.7 \cdot 10^{4}$ & $6 / 288$ & $2.08 \%$ & $1 / 6$ & 60 \\
\hline P8 & $5.7 \cdot 10^{3}$ & $5 / 288$ & $1.74 \%$ & $1 / 5$ & 20 \\
\hline P9 & $6.9 \cdot 10^{3}$ & $49 / 288$ & $17.01 \%$ & $49 / 49$ & $1.2 \cdot 10^{3}$ \\
\hline
\end{tabular}
predominantly Pseudomonas.

652 $b$ : Percentage refers to the number of isolates displaying a blue halo in relation to the 288 colonies tested. c: CFU/g soil of potential DAPG producers were calculated using the ratio of PCRconfirmed isolates compared to the total number of tested isolates times CFU/g soil of cultivable Pseudomonas. 


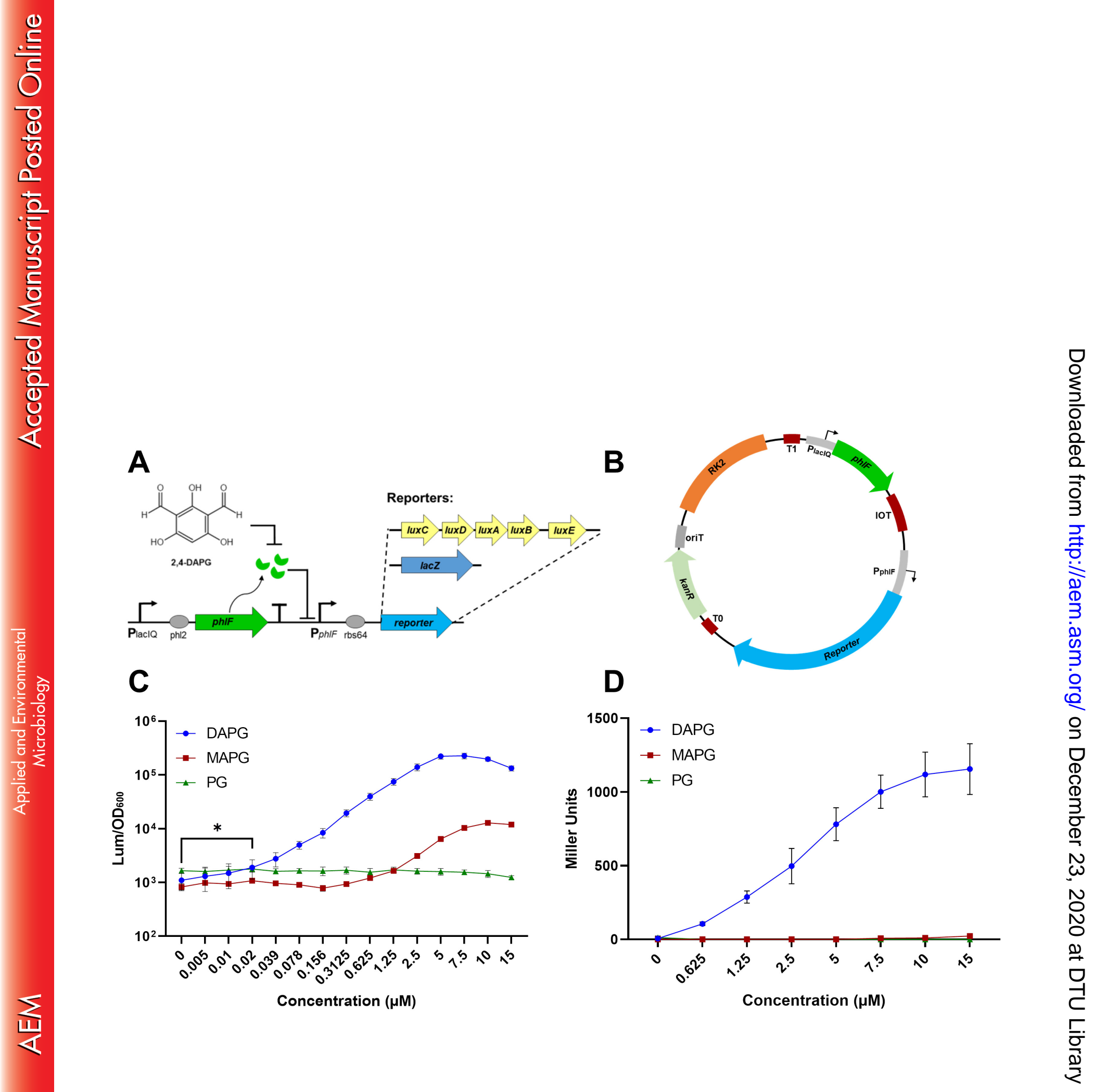




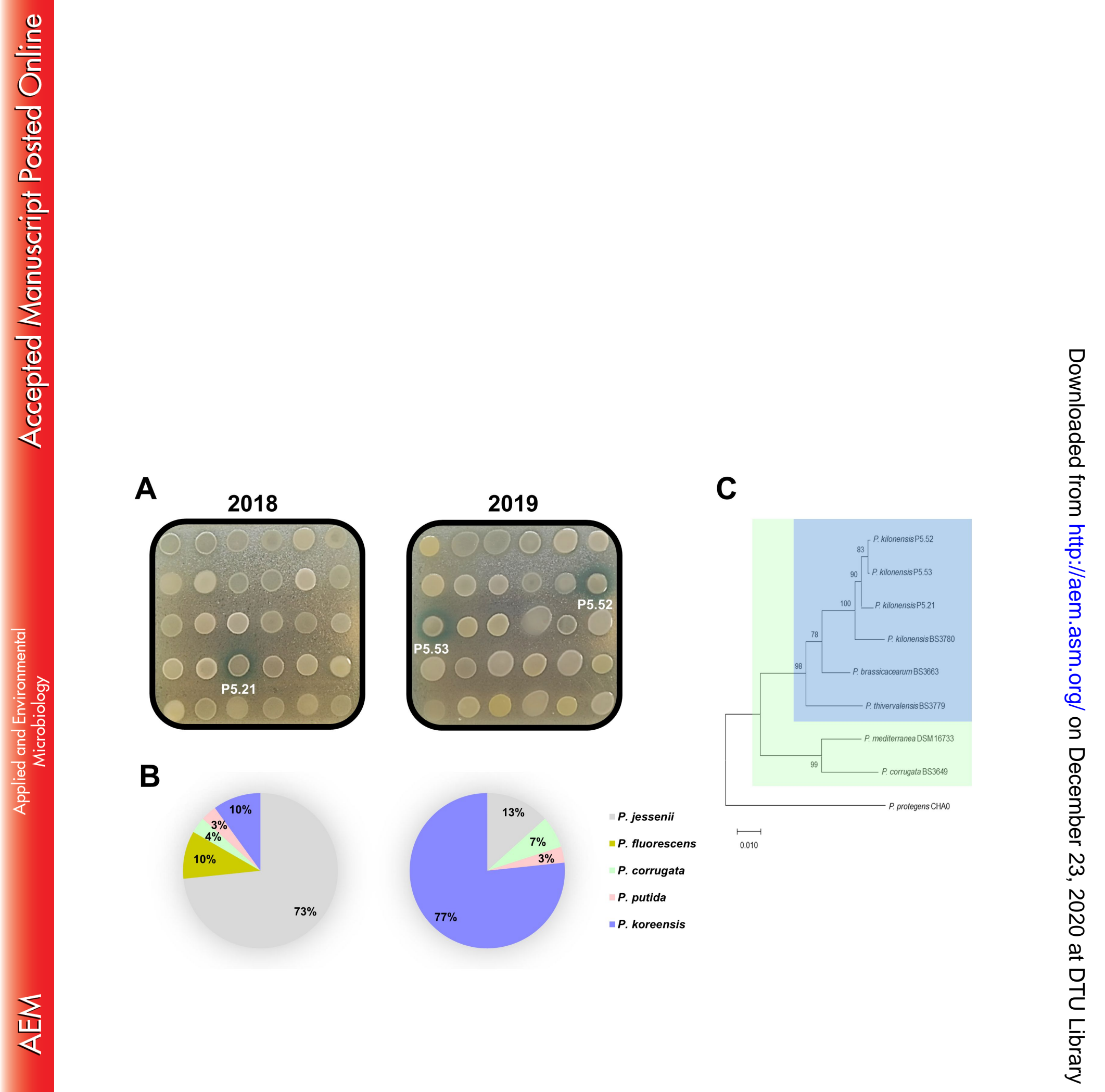


